\title{
Role of Fetal Umbilical Artery and Middle Cerebral Artery Doppler in Post Term Pregnancy and Neonatal Outcome
}

M.A.AbdElhai, M.A.Mohamed, M.A.Mahmoud and Y.Z.Gaber

Obstetrics \& Gynecology Dept., Faculty of Medicine, Benha Univ., Benha, Egypt

E-Mail:yous_only2004@yahoo.com

\begin{abstract}
The objective of the current study was to determine the value of fetal Doppler indices named middle cerebral artery (MCA)-PI, umbilical artery (UA)-PI and cerebro-placental ratio in pregnancies after 42 weeks gestational age and their correlation with the mode of delivery and perinatal outcome. This prospective, observational, clinical study was carried out on 150 women whose gestational age (GA) after 42 weeks' gestation from the first day of last normal menstrual period confirmed by early ultrasound examination during the first trimester attending the antenatal clinic of Benha University Hospital CerebroPlacenta Ratio with cutoff point 1.09 had the best specificity \&Middle Cerebral Artery Pulsatily Index with cutoff point $=1.01$ had the best sensitivity in prediction af adverse perinatal outcome in prolonged pregnancy . Cerebro-placental ratio shows a highest specificity $(93.76 \%) \%$ ) in comparison with middle cerebral artery and umbilical artery pulsatility indices( MCA-PI, UA-PI), so it may be a good test to reassure the obstetricians of the fetal wellbeing.
\end{abstract}

keywords: Post term pregnancy ,Umbilical artery Doppler,middle cerebral artery Doppler.

\section{Introduction}

Post-term pregnancy, by definition, refers to a pregnancy that has extended to or beyond 42 weeks of gestation (294 days, or estimated date of delivery [EDD] +14 days) [1].

The expressions post-term, postdate, postmature, \& prolonged are often loosely used interchangeably to signify pregnancies that have exceeded a duration considered to be the upper limit of normal [2].

The incidence of post term pregnancy ranges from $4 \%$ to $19 \%$ [3] .Post-term pregnancies are associated with numerous adverse outcomes. In addition to mortality, there is an increased risk of meconium aspiration syndrome, neonatal seizure and long term handicap [4].

Complications of prolonged pregnancy include instrumental delivery, macrosomia, meconium-stained amniotic fluid, oligohydramnios, increase rates of cesarean delivery, retained placenta, birth injury and perinatal deaths [5].

Fetal hypoxia is one of the major causes of high perinatal morbidity and mortality rates [6] The perinatal mortality rate (stillbirths plus early neonatal deaths) at 42 weeks of gestation is twice that at 40 weeks and increases 4 folds at 43 weeks [7].

Maternal-fetal risks increase from 41 weeks and starting antenatal testing at 41 weeks of gestation may decrease the complications [8].

In the surveillance of the uncomplicated prolonged pregnancy, Doppler information may play a role in differentiating which pregnancies may be followed by expectant management or determine whether induction is a better option [9].

Combining the Doppler waveform analysis of the middle cerebral artery (MCA) with that of the umbilical artery (UA) by a cerebroplacental ratio ( the ratio of their pulsatility indices) has been suggested as a useful clinical simplification [10].

The cerebro umiblical ratio shows a higher sensitivity $(80 \%)$ in predicting adverse prenatal outcome when compared with the UA S/D ratio $(56 \%)$ the UA PI (40\%) and the MCA PI (50\%) [11].

However, D'Antonio et al.,(2013)found that cerebroplacental ratio is not predictive of unfavorable outcome in women with pregnancies lasting more than 41 weeks [12].

\section{Material and methods}

150 women whose gestational age (GA) after 42 weeks' gestation from the first day of last normal menstrual period confirmed by early ultrasound examination during the first trimester attending the antenatal clinic of Benha University Hospital

were involved This prospective, observational, clinical study, conducted berween January2017 and July 2019.. Patients with Maternal medical disorders as: hypertension, diabetes mellitus, hepatic, cardiac, renal, autoimmune diseases (antiphospholipid syndrome, Systemic lupus erythematosus) \& anemia with pregnancy.Multiple pregnancy.Non-cephalic presentation.Premature rupture of membranes. Antepartum hemorrhage.In established labor. were excluded from the srudy.

All parienrs gave informed consent and the srudy prorocol was approved by the Hospital Research Ethics Committee.

$\begin{array}{ccc}\text { Adetailed } & \text { history } & \text { taking.Physical } \\ \text { examination.Routine } & \text { baseline } & \text { investigations. }\end{array}$

Ultrasound: Ultrasound and Doppler study had been done twice weekly till the onset of labor or termination of pregnancy in order to assess the following:

Gestational age determination.Fetal weight estimation.Placental site and grading.Fetal Biophysical profile scoring system . Aminotic fluid volum (oligohydraminos had been diagnosed if the largest vertical pocket $<2 \mathrm{~cm}$ ) Parameters of biophysical profile (fetal movements, fetal tone, fetal breathing and amniotic fluid volume) had been assessed

Doppler studies: was done to assess the following:Umbilical artery Doppler indices : umbilical 
artery pulsatility index (UA-PI), umblical artery resistence index (UA-RI) , umblical artery systolic/diastolic ratio (UA-S/D).Middle cerebral artery Doppler indices: middle cerebral artery pulsatility index (MCA-PI), middle cerebral artery resistence index (MCA-RI) ,middle cerebral artery systolic/diastolic ratio (MCA - S/D). Cerebro-placental ratio: had been calculated as a ratio of MCA-PI / UAPI .. Pulsed wave Doppler sonographic srudies were performed on women in Transabdominal ultrasound was performed to all patients while women in a slightly tilted position with the head of the bed raised 30 degrees \& a small pillow under the right loin. The ultrasound machine used was voluson 730 pro made in South Korea

\subsection{Umbilical artery doppler}

The patient was placed in a semi-recumbent position with a left lateral tilt, \& the uterine contents was scanned to select an area of amniotic cavity with several loops of umbilical cord. Ideally these cord loops should not be close to the cord insertion. Then using a pulsed wave Doppler on a free loop of cord, the characteristic sound \& shape of the umbilical artery identified.

When the screen show several waveforms of similar height, the image was frozen and the pulsatility index was estimated. A minimum of 3 separate reading were averaged before the final values obtained. Because of the potential effect of the fetal breathing movements on waveform variability, recording was performed during periods of fetal apnea . [13]

a) Middle cerebral artery Doppler:

An axial section of the brain, including the thalami and the sphenoid bone wings, should be obtained and Magnified. Color flow mapping should be used to identify the circle of Willis and the proximal MCA . The pulsed-wave Doppler gate should then be placed at the proximal third of theMCA, close to its origin in the internal carotid artery (the systolic velocity decreases with distance from the point of origin of this vessel). The angle between the ultrasound beam and the direction of blood flow should be kept as close as possible to $0^{\circ}$. Care should be taken to avoid any unnecessary pressure on the fetal head. At least three and fewer than 10 consecutive waveforms should be recorded. The highest point of the waveform is considered as the PSV(peak systolic velocity $(\mathrm{cm} / \mathrm{s})$.middle cerebral artery pulsatility index (MCA-PI) estimated

\subsection{Clinical management}

Spontaneous vaginal delivery was awaited, but induction of delivery was done occurred if there was oligohydraminos, biophysical profile is $\leq 6$

Induction of labor had done with Prostaglandin E2 vaginal suppositories, Oxytocin infusion or artificial amniotomy . With a Bishop score > 7, labor was induced by oxytocin infusion (starting with 5 IU in 500 $\mathrm{ml}$ Ringer solution \& the dose rate increased according to the response. \&/or artificial amniotomy. Otherwise, cervical ripening was performed with transvaginal prostaglandins (the suppository was placed in the posterior fornix then the patient was reassessed after 6 hours \& received another dose if required).

Fetal heart sound were auscultated every 15 minutes using fetal Doppler \& liquor was observed for meconium staining.

CTG was done for 20 minutes before the induction of labor for assessment of fetal wellbeing \& fitness for induction. CTG was repeated when needed during the induction for intrapartum assessment of fetal wellbeing $\&$ for early detection of intrapartum fetal distress. Fetal distress was detected by an abnormal CTG tracing. CTG tracing was considered abnormal when there was persistent late decelerations or persistent variable decelerations \& diminished FHR variability $(<5 \mathrm{bpm})$. These cases were terminated by CS.

\subsection{Perinatal outcome measures}

1. Apgar score at $1 \& 5$ minutes.

2. Assessment of neonatal birth weight.

3. Neonatal ICU admission.

4. Meconium aspiration syndrome.

\subsection{Adverse perinatal outcome}

Is defined as presence of one or more of the following conditions

- Cesarean delivery for fetal distress.

- Presence of thick meconium stained liquor.

- Apgar score $<7$ at 5 minutes.

- Meconium aspiration syndrome.

- Admission to neonatal intensive care unit (NICU).

\subsection{Statistics}

Atistics Comparison of all variables were done using by a software package SPSS 20.Sensitivity, Specificity, Positive predictive value, Negative predictive value of different arterial Doppler indices and cerebroplacental ratio (CPR) were evaluated. A receiver operator characteristic curve (ROC) was plotted to ascertain the best cut-off value of CPR for predicting adverse perinatal outcome in post-dated A probability value ( $\mathrm{p}$ value) more than 0.05 was considered to be not significant, $\mathrm{P}$ value less than 0.05 was considered to be statistically significant $\&$ p value less than 0.001 was considered to be statistically highly significant.

\section{Result}

Based on the presence or absence of adverse perinatal outcome, the study population was divided into two groups as follows:

\section{1st group:}

Group of adverse outcome was found to include 44 patients ( 24case cesarean section due to fetal distress, 4 cases neonatal ICU admission , 16 case meconium stained liquor, 3 cases meconium aspiration syndrome ) . 
2nd group:

Group of normal outcome was found to include 106

patients.

Table (1) Maternal_Demographic data of the 2 studied groups.

\begin{tabular}{lccc}
\hline Parameter & $\begin{array}{c}\text { Adverse perinatal } \\
\text { outcome }(\mathbf{n}=\mathbf{4 4})\end{array}$ & $\begin{array}{c}\text { Normal perinatal } \\
\text { outcome }(\mathbf{n}=\mathbf{1 0 6})\end{array}$ & P value \\
\hline $\begin{array}{l}\text { Maternal Age (years) } \\
\text { Mean } \pm \text { SD }\end{array}$ & $25.66 \pm 5$ & $25.51 \pm 3.14$ & 0.854 \\
Maternal Height (cm) & $160.77 \pm 1.91$ & $161.12 \pm 4.45$ & 0.655 \\
Mean \pm SD & $78.19 \pm 6.09$ & $76.98 \pm 6.69$ & 0.372 \\
Maternal Weight (Kg) & & & \\
Mean \pm SD & $293.13 \pm 38.29$ & $290.3 \pm 34.85$ & 0.708 \\
GA at delivery (days) & $1.17 \pm 1.59$ & $1.12 \pm 1.55$ & 0.878 \\
Mean \pm SD & $3(1-8)$ & $3.5(1-6)$ & 1.000 \\
$\begin{array}{l}\text { Gravidity } \\
\text { Median\&range }\end{array}$ & $0.58 \pm 0.87$ & $0.58 \pm 0.81$ & \\
Parity & $3.5(0-7)$ & $2.5(0-5)$ & \\
Median\&range & & & \\
\hline
\end{tabular}

3.1 Demographic data

This table shows no statistically significant difference between the 2 groups by using unpaired $\mathrm{t}$ test as regards the maternal age, height, weight,

gravidity, parity \& gestational age $(\mathrm{P}>0.05)$.

3.2 Doppler indices

Table (2) Comparison between both groups as regards: UA-PI, MCA-PI, MCA PI: UA PI ratio.

\begin{tabular}{lccc}
\hline Parameter & $\begin{array}{c}\text { Adverse perinatal } \\
\text { outcome }(\mathbf{n}=\mathbf{4 4})\end{array}$ & $\begin{array}{c}\text { Normal perinatal } \\
\text { outcome }(\mathbf{n = 1 0 6})\end{array}$ & P value \\
\hline Mean UA- PI & $0.99 \pm 0.17$ & $0.86 \pm 0.2$ & 0.002 \\
Mean MCA- PI & $0.95 \pm 0.2$ & $1.28 \pm 0.28$ & 0.004 \\
Mean MCA-PI: UA PI ratio & $0.96 \pm 0.16$ & $1.48 \pm 0.22$ & 0.004 \\
\hline
\end{tabular}

Data were presented as mean \pm standard deviation.

This table shows that adverse outcome group had higher UA-PI but had a lower MCA-PI \& MCA-PI: UA-PI ratio when compared to the normal outcome group with statistically highly significant differences between both groups by using unpaired t-test $(\mathrm{p}<$ $0.001)$.

\subsection{Mode of delivery}

Table (3) Comparison between both groups as regards the mode of delivery and the indication of CS .

\begin{tabular}{|c|c|c|c|c|}
\hline \multicolumn{2}{|l|}{ Parameter } & $\begin{array}{c}\text { Adverse perinatal } \\
\text { outcome }(n=44)\end{array}$ & $\begin{array}{l}\text { Normal perinatal } \\
\text { outcome }(n=106)\end{array}$ & $P$ value \\
\hline \multicolumn{2}{|c|}{ Spontaneous VD } & $5(11.36 \%)$ & $50(47.17 \%)$ & 0.003 \\
\hline \multicolumn{2}{|c|}{ Induced VD } & $15(34.1 \%)$ & $25(23.58 \%)$ & 0.285 \\
\hline \multicolumn{2}{|l|}{ CS } & $24(54.54)$ & $31(29.25 \%)$ & 0.01 \\
\hline \multirow[t]{2}{*}{ Indication } & Failed induction & $6(25.0 \%)$ & $10(32.26 \%)$ & 0.648 \\
\hline & & $2(8.34 \%)$ & $18(58.07 \%)$ & 0.002 \\
\hline \multirow[t]{2}{*}{ Of CS } & Failure of progress & (inductioin) & $\begin{array}{l}12 \text { case induction } \\
6 \text { cases spontaneous }\end{array}$ & \\
\hline & Fetal distress & $16(66.66 \%)$ & $3(9.67 \%)$ & 0.0004 \\
\hline
\end{tabular}

Data were presented as number $(\%)$.

This table shows that the adverse outcome group had a higher rate of Cesarean section compared to the normal outcome group with statistically highly significant difference between both groups $(\mathrm{p}<0.001)$.
Also This table shows that the group of adverse outcome had a higher rate of CS due to fetal distress compared to the other indications of CS with statistically highly significant differences between both 
groups by using chi-square test $(\mathrm{p}<0.001)$.

Neonatal birth weight and its relation to perinatal outcome

Table (4) Comparison between both groups as regards the neonatal birth weight

\begin{tabular}{lccc}
\hline Parameter & Adverse & Normal & P valu \\
\hline & perinatal & perinatal outcome & e \\
& outcome $(\mathbf{n}=\mathbf{4 4})$ & $\mathbf{n}=\mathbf{1 0 6})$ & \\
Neonatal & $2775.83 \pm$ & $3251.9 \pm 204.94$ & 0.0001 \\
birthweight & 166.15 & & \\
\hline
\end{tabular}

Data were presented as mean \pm standard deviation.

This table shows highly statistically significant difference as regards the neonatal birth weight between

both groups by using unpaired t-test. $(\mathrm{p}<0.001)$.

\section{Umbilical artery PI}

Table (5) Relation between umbilical artery PI \& perinatal outcome \& the mode of delivery on considering the cutoff point $=1.18($ no. \& \%)

\begin{tabular}{lccc}
\hline Parameter & $\begin{array}{c}\text { UA-PI }<\mathbf{1 . 1 8} \\
(\mathbf{n = 1 1 8})\end{array}$ & $\begin{array}{c}\text { UA-PI } \geq \\
\mathbf{1 . 1 8}(\mathbf{n}=\mathbf{3 2})\end{array}$ & P-value \\
\hline Vaginal delivery & $78(66.1 \%)$ & $17(53.13 \%)$ & \\
CS for fetal distress & $11(9.32 \%)$ & $8(25 \%)$ & 0.1074 \\
Other indications of CS & $29(24.58 \%)$ & $7(21.87 \%)$ & \\
Total no of adverse outcome & $26(22.03 \%)$ & $18(56.25 \%)$ & 0.01 \\
Total no of normal outcome & $92(77.97 \%)$ & $14(43.75 \%)$ & \\
\hline
\end{tabular}

Data were presented as number $(\%)$. UA-PI = umbilical artery pulsatility index.

This table shows that the rate of CS for fetal distress was higher in the abnormal group(UA-PI >1.18) compared to the normal group(UA-PI <1.18). However, the difference was not statistically significant $(\mathrm{p}>0.05) \&$ the total number of patients who had adverse perinatal outcome was higher among the abnormal group compared to the normal group with statistically significant differences between both groups by using chi-square test. $(\mathrm{p}<0.05)$.

\section{Middle cerebral artery $\mathbf{P I}$}

Table (6) Relation between middle cerebral artery PI \& perinatal outcome \& the mode of delivery on considering the cutoff point $=1.01($ no. $\& \%)$.

\begin{tabular}{lccc}
\hline Parameter & $\begin{array}{c}\text { MCA-PI } \leq \\
\mathbf{1 . 0 1}(\mathbf{n = 9 2})\end{array}$ & $\begin{array}{c}\text { MCA-PI > } \\
\mathbf{1 . 0 1}(\mathbf{n = 5 8})\end{array}$ & P-value \\
\hline Vaginal delivery & $62(67.4 \%)$ & $33(56,89 \%)$ & \\
& $15(16.3 \%)$ & $4(6.89 \%)$ & 0.039 \\
CS for fetal distress & $15(16.3 \%)$ & $21(36.22 \%)$ & \\
Other indications of CS & $35(38.04 \%)$ & $9(15.52 \%)$ & 0.004 \\
Total no of adverse outcome & $57(61.96 \%)$ & $49(84.48 \%)$ & \\
Total no of normal outcome & & & \\
\hline
\end{tabular}

Data were presented as number $(\%) . \mathrm{MCA}-\mathrm{PI}=$ middle cerebral artery pulsatility index.

This table shows that the rate of CS for fetal distress was higher in the abnormal group (MCA-PI $<1.01)$ compared to the normal group (MCA-PI >1.01) with statistically significant differences between both groups $(\mathrm{P}<0.05) \&$ the total number of patients who had adverse perinatal outcome was higher among the abnormal group compared to the normal group with statistically highly significant differences between both groups by using chi-square test. $(\mathrm{p}<0.001)$. 


\section{Cerebroplacental ratio}

Table (7) Relation between MCA-PI/UA-PI ratio (CPR) \& perinatal outcome \& the mode of delivery on considering the cutoff point $=1.09($ no. $\& \%)$ :

\begin{tabular}{lccc}
\hline Parameter & $\begin{array}{c}\text { CPR }>\mathbf{1 . 0 9} \\
(\mathbf{n = 1 0 3})\end{array}$ & $\begin{array}{c}\mathbf{C P R} \leq \mathbf{1 . 0 9} \\
(\mathbf{n}=\mathbf{4 7})\end{array}$ & P value \\
\hline Vaginal delivery & $68(66.02 \%)$ & $27(57.45 \%)$ & \\
CS for fetal distress & $6(5.83 \%)$ & $13(27.66 \%)$ & 0.0003 \\
Other indications of CS & $29(28.15 \%)$ & $7(14.89 \%)$ & \\
Total no of adverse outcome & $10(9.71 \%)$ & $34(72.34 \%)$ & 0.0001 \\
Total no of normal outcome & $93(90.29 \%)$ & $13(27.66 \%)$ & \\
\hline
\end{tabular}

Data were presented as number $(\%) . \mathrm{CPR}=$ cerebro-placental ratio.

This table shows that the rate of CS for fetal distress was higher in the abnormal group $(\mathrm{CPR}<1.09)$ compared to the normal group $(\mathrm{CPR}>1.09)$ with highly statistically significant differences between both groups $(\mathrm{P}<0.001) \&$ the total number of patients who had adverse perinatal outcome was higher among the abnormal group compared to the normal group with highly statistically significant differences between both groups by using chi-square test. $(\mathrm{P}<0.001)$.

\section{Significance Tests}

Table (8) It shows the performance of the different testing parameters

\begin{tabular}{lcccccc}
\hline Parameter & $\begin{array}{c}\text { Area } \\
\text { under } \\
\text { curve }\end{array}$ & $\begin{array}{c}\text { Cut off } \\
\text { value }\end{array}$ & Sensitivity & Specificity & PPV & NPV \\
\hline $\begin{array}{l}\text { Umbilical } \\
\text { PI }\end{array}$ & 0.507 & 1.184 & $36.31 \%$ & $65.93 \%$ & $59.19 \%$ & $70.54 \%$ \\
\hline MCA PI & 0.649 & 1.012 & $80.46 \%$ & $48.48 \%$ & $46.76 \%$ & $81.86 \%$ \\
\hline CPR & 0.925 & 1.093 & $75.1 \%$ & $93.76 \%$ & $87.17 \%$ & $86.97 \%$ \\
\hline
\end{tabular}

This table shows comparison between the performances of the different Dopper indices in antepartum fetal surveillance for adverse perinatal

\section{Discussion}

prolonged pregnancy is associated with adverse outcomes like fetal distress, meconium aspiration syndrome and more neonatal ICU admissions. The outcome of prolonged pregnancy can be improved by proper counselling for follow up during pregnancy and proper monitoring and appropriate management during labour [14].

There is insufficient evidence to recommend a management strategy between 40 and 42 completed weeks. Thus, although not considered mandatory, initiation of fetal surveillance at 41 weeks is a reasonable option. After completing 42 weeks, recommendations are for either antenatal testing or labor induction [15].

The present study included 150 pregnant ladies who were divided into two groups based on presence or absence of adverse perinatal outcome. The group of adverse outcome included 44 patients and the group of normal outcome included 106 patients.

All the women were subjected to ultrasonographic evaluation of the Doppler velocimetry study of umbilical \& middle cerebral artery. outcome. It was found that CPR with cutoff point 1.09 had the best specificity \&MCA PI with cutoff point = 1.01 had the best sensitivity.

It was noted that there were no recorded cases of stillbirth or neonatal death among the studied population.

Results of current study showed no statistically significant difference regarding maternal age between the two groups. This result agrees with [16]. [17] found that a negative effect of maternal age on the neonatal outcome may be seen even after 30 years of age. [18] found that pregnancies of maternal age $\geq 40$ years carry increased risks for both neonatal and obstetric complications, and these risks seem to be effected by parity

Results of the present study showed no statistically significant difference regarding maternal body weight between the two studied groups. However Bali Ram etal.,(2018) [19] found that Maternal BMI have strong association with pregnancy complications and outcome.

The findings of current study showed no statistically significant differences regarding parity \& gravidity between the two groups. These results agree with Yun Wang et al., (2010) [20] who found that there is no significant effect on adverse perinatal outcome irrespective of parity. But Singh \& Sankaran et al., 
(2008) [21] found that nulliparity was associated with increased risk of adverse intrapartum event.

Results of the present study showed that there was statistically significant difference between the two groups as regard the fetal birth weight which is lower in the group of adverse outcome. These results agree with [21] who found that the fetal birth weight was significantly less in the group with adverse events.

But it differs from the results of [22] that reported that the fetal weight seems to be a poor predictor of the outcome of prolonged pregnancy.

Results of current study had shown that the rate of cesarean section among all patients was $37 \%, \&$ the rate of vaginal delivery among all patients was $63 \%$. This disagrees with [14] who documented increased cesarean section rates in case of prolonged pregnancy.

By analysis of data in the two studied groups regarding the mode of delivery, $11.36 \% \%$ of patients who had adverse outcome \& $47.17 \%$ of patients that had normal outcome were delivered by spontaneous vaginal delivery. While $34.1 \%$ of patients in the group I \& $23.58 \%$ in the group II were delivered by induced vaginal delivery . Also, $54.54 \%$ of patients in the group I \& $29.25 \%$ in the group II were delivered by cesarean section. Regarding the cause of CS in the two groups $25.0 \%$ due to failed induction, $8.34 \%$ due to failure of progress \& $66.66 \%$ due to fetal distress in the group I, but in the group II $32.26 \%$ due to failed induction \& $58.07 \%$ due to failure of progress, $9.67 \%$ due to fetal distress. There were highly significant differences between the two studied groups regarding the mode of delivery.

These results agree with those reported by [23] who found highly significant difference between postdate and term groups as regard delivery by CS.

Induction of labour in late and post term pregnancies is associated with a significantly higher cesarean section rate. Other maternal and fetal parameters were not influenced by induction of labour [24 ].

Also, [25] showed that Cesarean section was significantly more common in women with post-term pregnancy. The increase was equally distributed between Cesarean sections performed for failure to progress in labor and fetal distress. This finding is consistent with the hypothesis that some cases of prolonged pregnancy are associated with a defect in the physiology of labor, in addition to any increase in risk of fetal hypoxia. However, the possibility of bias in management arising out of the knowledge that a pregnancy is post-term cannot be excluded as a factor in the increase in Cesarean section rates.

Absent or reversed end diastolic flows indicate marked placental resistance $\&$ has been associated with fetal deterioration \& increased risk of perinatal mortality \& operative delivery [26].

Frequency of delivery by caesarean section was higher in foetuses with abnormal Doppler. Incidence of meconium stained liquor and intra-partum death was higher $(50 \%)$ in reversed end diastolic flow [27].
In the current study, no case with absent or reversed end diastolic flow was recorded.

Regarding the umbilical artery pulsatility index, the patients with abnormal UA-PI (with values above the cutoff point) had Statistically significant difference with those who had normal UA-PI as regard the incidence of adverse perinatal outcome $(p<0.05)$ but there was statistically non significant difference between both groups as regard mode of delivery $(\mathrm{p}>0.05)$.

On considering the cutoff point of 1.18 for umbilical artery pulsatility index, operative interference due to fetal distress \& presence of adverse outcome were found to be higher in the abnormal group compared to the normal group. This differs from the results reported by (28) who found that the average UA-PI for the low risk pregnancies was 0.92 .

The results of the present study agree with those reported by [29] who found that UA-PI may be useful in assessment of FHR patterns suggesting fetal distress.

On the other hand, [30] reported that UA-PI was found to be significantly lower in cases of prolonged pregnancy with fetal distress. Also, [31] showed that fetuses that had some degree of distress had UA-PI within normal range. [32] showed that abnormal flow velocity had no significant relationship with fetal asphyxia, indicating that the structural changes of placenta during prolonged pregnancy didn't alter fetal blood flow. [33] reported that UA velocimetry is a test of placental function that does not always directly reflect fetal status.

[34] found that UA Doppler flow velocity waveform from pregnancies associated with fetal compromise \& abnormal neonatal outcome were similar to those from pregnancies in which outcome was normal.

Vascular resistance in the umbilical artery and middle cerebral artery does not change abruptly when gestation exceeds 280 days. It also cannot be taken as the sole method of fetal surveillance when date is crossed [35].

But CPI ratio is a very good predictor of adverse outcome in the fetuses of women with high-risk pregnancy [36].

Evaluation of performance of the UA-PI assessment test in the current study revealed that it had a sensitivity of $36.31 \%$ \& specificity of $65.93 \%$. [37] reported that the sensitivity of UA Doppler velocimetry was about $64 \%$.

Hypoxia is present in most of the chronic or acute fetal patency stages. The initial cardiovascular response includes a redistribution of the main fetal flows and later blood pressure increase, bradycardia, and modification of the cardiac (left and right) hemodynamics. It is generally accepted that there is a significant vasoconstriction of most of the fetal vessels (pulmonary, splanchnic, skeletal, and muscular areas) and an increased perfusion of the brain, the heart, and the adrenal glands. This phenomenon, called the "brain-sparing effect " [38]. 
This phenomenon, "brain-sparing," is actually a misnomer, as it is not protective for the fetus but rather is associated with perinatal morbidity and mortality [39].

Regarding the middle cerebral artery pulsatility index, the patients with abnormal MCA-PI (with values below the cutoff point) had Statistically significant difference with those who had normal MCA-PI as regard the incidence of adverse perinatal outcome \& risk of cesarean delivery especially for fetal distress $(\mathrm{p}<0.05)$.

On considering the cutoff point of 1.01 for middle cerebral artery pulsatility index, cesarean delivery due to fetal distress was found to be significantly higher in the abnormal group than in the normal group.

[40] found that MCA-PI is better than UA-PI \& amniotic fluid volume assessment to predict the adverse outcome in uncomplicated postdated pregnancy. Also, [41] showed that women who developed signs of fetal distress during labor or who required intervention because of fetal distress had decreased MCA-PI.

However, [42] discovered absence of any significant statistical difference in UA-PI \& MCA-PI in predicting adverse perinatal outcome. They attributed this to that in uncomplicated postdate pregnancies; the mechanism of fetal compromise is perhaps due to decrease in the flow of nutrients across the placenta $\&$ decrease in the efficiency of utilization of nutrients by the placenta $\&$ the fetus. These changes are not reflected in increase in resistance in the blood vessels of placenta or fetus.

Also, [43] found that MCA-PI is not a reliable indicator for predicting fetal distress.

The CPR index shows the highest sensitivity in prediction of FHR abnormalities and adverse neonatal outcome in uncomplicated pregnancies at 40 weeks and beyond. The CPR index is useful in clinical practice in antenatal monitoring of these women in order to select those at high risk of intra- and postpartum complications [44].

Evaluation of performance of the MCA-PI assessment test in the current study revealed that it had a sensitivity of $80.46 \%$ \& specificity of $48.48 \%$.

Regarding the cerebro-placental ratio (MCAPI/UA-PI), the patients that had abnormal ratios (with values below the cutoff point, on considering the cutoff point of 1.09 ) were compared with the cases that had normal values regarding the incidence of adverse perinatal outcome $\&$ risk of cesarean delivery especially for fetal distress, highly significant differences were found between the two studied groups $(\mathrm{p}<0.01)$.

These results agree with [45], where PI was determined in the mid-portion of the umbilical artery \& the mid or distal segment of the middle cerebral artery, the CPR offers the advantage of detecting redistribution of blood flow due to two potential mechanisms: either "forced centralization" an increase of the placental resistance or decrease in the cerebral blood flow due to a "brain-sparing" effect, accordingly CPR considered better predictor of fetal compromise than either vessel considered alone.

Similarly, [46] , [47] , [48] support these results regarding CPR. They found that fetuses may undergo redistribution of arterial blood flow in response to a failing of uteroplacental unit before marked changes occur in the umbilical artery waveform. So, CPR is a better predictor of fetal compromise than neither MCAPI nor UA-PI alone.

However, [49] in their study on UA-PI, MCA-PI \& CPR in postdated pregnancies concluded that Doppler information may play a role in differentiating postdated pregnancies which may be followed by expectant management from those in whom induction is a better option. However, low positive predictive value of CPR can result in missing cases with fetal jeopardy \& high false positive rate can result in undue concern, expensive tests \& unnecessary interference.

Also, [50] found that CPR can predict adverse outcome only in fetuses $<34$ weeks gestation.

Evaluation of performance of the CPR assessment test in the current study revealed that it had sensitivity of $75.1 \%$ \& specificity of $93.76 \%$ on considering the cutoff point of 1.09 . These results agree with [51] who found that cutoff point 1.05 can predict the adverse perinatal outcome with sensitivity of $80 \% \&$ specificity of $95 \%$. In contrast, Gramellini [52]\& [43] used a cutoff value of 1.08 which had a higher sensitivity \& specificity when compared with UA-PI \& MCA-PI . [53] found that CPR with cutoff value of 1.1 had $62 \%$ sensitivity \& $74.5 \%$ specificity to predict adverse outcome. [54] used a cutoff value of 1.0 which had a higher sensitivity \& specificity to predict the adverse outcome.

[12] concluded that cerebroplacental ratio is not predictive of unfavorable outcome in women with pregnancies lasting more than 41 weeks by using 0.98 as a cutoff value.

Although CU ratio of 1.3 assures the obstetrician of fetal well being, its low specificity and high false positive value can lead to unnecessary tests and intervention. Hence it is not an ideal test for routine antepartum fetal surveillance in low risk postdate pregnancies [55].

Results of the present study showed that CPR had highest specificity $(93.76 \%)$ in comparison with other parameters . On the other hand, MCA-PI had highest sensitivity $(80.46 \%) \%)$ in comparison with other parameters followed by CPR (75\%).

\section{Conclusion}

Doppler information may play a role in differentiating which pregnancies may be followed by expectant management or determine whether induction of labor is a better opinion.

Cerebro-placental ratio shows a highest specificity $(93.76 \%) \%$ ) in comparison with middle cerebral artery and umbilical artery pulsatility indices( MCA-PI, UAPI), so it may be a good test to reassure the 
obstetricians of the fetal wellbeing .However further studies on large number of cases will be needed to assertion this study

\section{Referance}

[1] American College of Obstetricians and Gynecologists, Management of intrapartum fetal heart rate tracings. Practice Bulletin,Vol.69,PP.116,2013.

[2] F.Cunningham , K.J.Leveno, S.L.Bloom, C.Y.Spong, J.S.Dashe, B.L. Hoffman, Fetal assessment. In Williams Obstetrics.Vol.24 ${ }^{\text {th }} \mathrm{ed}$, Ch.17, PP. 335-348, 2014

[3] M.Y.Divon \& N.Feldman-Leidner, Postdatesand ante natal testing. Semin Perinatal.Vol. 32 (4),PP. 295,2008.

[4] K.Maryam, I.Shahrokh \& M.Masoome, The predictive value of ultrasound assessment of amniotic fluid index, biophysical profile score, nonstress test and foetal movement chart for meconium,Vol.39,PP.225-230,2014.

[5] A.W. Olesen, J.G.Westergaard \& J.Olsen, Perinatal and maternal complication related to postterm delivery: a national register-based study. 1978-1993. Am J Obstet Gynecol. Vol. 189,PP. 222-227, 2003.

[6] T.Korbelak, M.Ropacka-Lesiak \& G.Bręborowicz, Doppler blood flow velocimetry in the umbilical artery in uncomplicated pregnancy Vol. 83,PP.38-45, 2012.

[7] E.R.Norwitz, V.V. Snegovskikh \& A.B.Caughey, Prolonged pregnancy: when should we intervene? Clin Obstet Gynecol.Vol. 50,PP. 547-557, 2007.

[8] J.Zeynep \& I.Gumus, Prolonged Pregnancies: Approaches in Clinical Management, The New Journal of Medicine.Vol. 26,PP. 11-15, 2009.

[9] F.Figueras, M.Lanna \& M.Palacio, Middle cerebral artery Doppler indices at different sites: prediction of umbilical cord gases in prolonged pregnancies. Ultrasound Obstet Gynecol.Vol. 24,PP.529-533, 2004.

[10] C.Ebbing, S.Rasmussen \& T.Kiserud, Middle cerebral artery blood flow velocities and pulsatility index and the cerebroplacental pulsatility ratio: longitudinal reference ranges and terms for serial measurements. Ultrasound Obstet Gynecol.Vol. 30,PP.287-296, 2007.

[11] M.El-Sokkary, M.Omran \& H.Ahmed, Ratio of Middle Cerebral Artery/Umbilical Artery Doppler Velocimetry and Status of Newborn in Postterm Pregnancy. Journal of American Science.Vol. 7(4),PP. 542-549, 2011.

[12] F.D'antonio, D.Patel, N.Chandrasekharan, B.Thilaganathan \& A.Bhide, Role of cerebroplacental ratio for fetal assessment in prolonged pregnancy Ultrasound Obstet Gynecol.Vol.42(2),PP. 196-200, 2013.

[13] A.Bhide, G.Acharya, C.Bilardo, ISUOG Practice Guidelines: use of Doppler ultrasonography in obstetrics. Ultrasound Obstet
Gynecol. Vol. 41,PP. 233-239,2013.

[14] Vandana Verma, Vaibhav Kanti, Pragya Shree, Maternal and fetal outcome in post term pregnancy. International Journal of Reproduction, Contraception, Obstetrics and Gynecology (IJRCOG), Vol. 6, PP.188-190, 2017.

[15] F.Cunningham, K.J.Leveno, S.L.Bloom, C.Y.Spong, J.S.Dashe, B.L.Hoffman, Fetal assessment. In Williams Obstetrics, $24^{\text {th }}$ ed, Ch.Vol.17, PP. 335-348, 2014.

[16] R.Matijeviae, Outcome of post-term pregnancy: A matched-pair Case-Control Study. Croatian Medical J., Vol. 39(4),PP. 55-61, 1998.

[17] G,Usha, C.Suhasini \& M.K.Narula, Value of middle cerebral artery to umbilical artery ratio by Doppler velocimetry in pregnancies beyond term. J Obstet Gynecol India.Vol. 56(1),PP. 37-40, 2006.

[18] Marie Blomberg, Rasmus Birch Tyrberg \&Preben Kjølhed, Impact of maternal age on obstetric and neonatal outcome with emphasis on primiparous adolescents and older women: a Swedish Medical Birth Register Study,Vol.4, PP.25, 2012.

[19] B.Eralp , D.Kerem , E.Selçuk , F.Mehmet , Y.İlkin Mahmut , K.Oktay , Ç.Turhan , and D.Nuri , The impact of parity on perinatal outcomes in pregnancies complicated by advanced maternal age. The impact of parity on perinatal outcomes in pregnancies complicated by advanced maternal age.J Turk Ger Gynecol Assoc.Vol. 14 (4),PP. 205-209, 2013.

[20] Bali Ram Sharma, Reena Sharma, Poojan Dogra, A prospective study on effect of maternal BMI on fetal outcome . Int J Reprod Contracept Obstet Gynecol Jul,Vol.7(7),PP. 2782-2785,2018.

[21] W.Yun \& T.Torn, The impact of advanced maternal age \& parity on obstetric \& perinatal outcome in singleton gestation. Arch Obstet Gynecol. Vol. 284(1),PP. 31-37, 2011.

[22] R.O.Bahado-Singh , A.U.Oz , C.Hsu , E.Kovanci , O.Deren , L.Onderoglu , G. Mari ,Middle cerebral artery Doppler velocimetric deceleration as a predictor of fetal anemia in $\mathrm{RH}$ alloimmunized fetuses without hydrops. Am J Obstet Gynecol.Vol. 183,PP.746, 2000.

[23] T.Singh, S.Sankaran , B.Thilaganathan \& A.Bhide, The prediction of intra-partum fetal compromise in prolonged pregnancy. J Obstet Gynecol.Vol. 28(8),PP. 779-782, 2008.

[24] K.Rasmussen \& A.Aggar, Influence of fetal weight on outcome of prolonged pregnancy. Ugeskrift for Leager.Vol. 162(32),PP. 4265 4267, 2000.

[25] M.Tasic, V.Lilic \& M.Stefanovic, Placental insufficiency in pregnancy after $40^{\text {th }}$ week of gestation. Acta Medica Medianae.Vol. 46,PP.2629, 2007. 
[26] F.Thangarajah, P.Scheufen, V.Kirn, \& P.Mallmann, Induction of Labour in Late and Postterm Pregnancies and its Impact on Maternal and Neonatal Outcome Geburtshilfe Frauenheilkd.Vol.76(7),PP. 793-798, Jul ,2016.

[27] M.Luckas, W.Buckett \& Z.Alfirevic, Comparison of outcomes in uncomplicated term and post-term pregnancyfollowing spontaneous labor. J Perinat Med.Vol. 26,PP. 475-479, 1998.

[28] B.J.Trudinger, C.M.Cook \& M.A.Jonsel, A comparison of fetal heart rate monitoring \& umbilical artery waveforms in the recognition of fetal compromise. $\mathrm{Br} \mathrm{J}$ Obstet Gynecol.Vol. 93,PP.171-175, 2002.

[29] V.Shiny, K.Gagandeep, M.Kanika \& D.Tapasya, Abnormal umbilical artery Doppler in third trimester and perinatal outcome: a retrospective study. International Journal of Reproduction, Contraception, Obstetrics and Gynecology Varghese $S$ et al. Int J Reprod Contracept Obstet Gynecol.Vol. 5(7),PP. 21962199, Jul ,2016.

[30] G.G.Romero, A.L.Ponce \& P.S.Ramos, Doppler flowmetric fetal indices in low risk pregnancies. Obstet Gynecol Mex.Vol. 67,PP.484-490, 2002.

[31] D.Gunyemi , R.Stanley , C.Lynch , D.Edwards , T.Fukushima , Umbilical artery velocimetry in predicting perinatal outcome with intrapartum fetal distress. ACOG. Vol. 80(3),PP. part 2, 1992.

[32] P.Olofsson, P.Saldeen \& K.Marsal, Association between a low umbilical artery pulsatility index \& fetal distress in labor in very prolonged pregnancies. Eur J Obstet Gynecol Repord Biol.Vol. 73(1),PP. 23-29, 2000.

[33] P.Zimmerman, T.Alback, J.Kiskinen , R.Vaalamo, R.Tui- T.mala, Ranta, Doppler flow velocimetry of the umbilical artery, uteroplacental arteries and fetal middle cerebral artery in prolonged pregnancy. Ultra- sound Obstet Gynecol.Vol. 5,PP.189-192, 1995.

[34] P.Malcus, K.Marsal \& P.H.Persson: Fetal \& uteroplacental blood flow in prolonged pregnancies, a clinical study. Ultrasound Obstet Gynecol.Vol. 1,PP.40-45, 1991.

[35] F.Figueras, M.Lanna \& M.Palacio, Middle cerebral artery Doppler indices at different sites: prediction of umbilical cord gases in prolonged pregnancies. Ultrasound Obstet Gynecol.Vol.24,PP.529-533, 2004.

[36] H.J. Stokes, R.V.Roberts \& J.P.Newnham, Doppler flow velocity waveform analysis in postdate pregnancies. Aust N Z J Obstet Gynecol.Vol. 31(1),PP. 27-30, 2008.

[37] G.Subhra, M.Kirtirekha, S.Sanjukta, N.Pallabi, Study of Doppler indices of umbilical artery and middle cerebral artery in pregnancies at and beyond forty weeks of gestation. Vol. 5, PP.12, 2016
[38] D.Ramkanwar \& G.Nikhila, The value of the middle cerebral to umbilical artery Doppler ratio in the prediction of neonatal outcome in patients with high risk pregnancy. International Journal of Reproduction, Contraception, Obstetrics and Gynecology.Vol.6(9), PP. 3965-3968, ,2017.

[39] T.Dubinsky, M.Lau, O.Thompson, J.Garcia JMastobattista, Predicting poor perinatal outcome: A comparative study of noninvasive antenatal testing methods. Am J Roentgenol. Vol. 186(3),PP. 827-831, 2000.

[40] P.Arbeille, F.Perrotin \& A.Salihagić, Fetal Doppler hypoxic index for the prediction of abnormal fetal heart rate at delivery in chronic fetal distress. Eur J Obstet Gynecol Reprod Biol.Vol. 121,PP.171-177,2005.

[41] R.Cruz-Martinez, F.Figueras, E.HernandezAndrade, Fetal brain Doppler to predict cesarean delivery for nonreassuring fetal status in term small-for-gestational-age fetuses. Obstet Gynecol.Vol. 117,PP.618, 2011.

[42] H.Lam, W.C. Leung \& T.T.Lao, The use of fetal Doppler cerebroplacental blood flow \& amniotic fluid volume measurement in the surveillance of postdated pregnancies. Acta Obstet Gynecol Scand.Vol.84,PP.844-848, 2005.

[43]

Y.Eyal \& T.Ofen, Post-term pregnancies with normal cardiotocography \& amniotic fluid columns: the role of Doppler evaluation in predicting perinatal outcome. Er J Obstet Gynecol.Vol. 54(2),PP. 93-98, 1994.

[44] G.Usha, C.Suhasini \& M.K.Narula, Value of middle cerebral artery to umbilical artery ratio by Doppler velocimetry in pregnancies beyond term. J Obstet Gynecol India. Vol. 56(1),PP. 37-40, 2006.

[45] B.Shahina \& V.Mehta, Color Doppler evaluation of cerebroplacental ratio \& its usefulness in diagnosis of intrauterine growth restriction \& prediction of adverse perinatal outcome. Ind J Radiol, Vol. 20(1),PP. 20-25, 2010.

[46] Mariola Ropacka-Lesiak, Tomasz Korbelak, Joanna Świder-Musielak, and Grzegorz Breborowicz, Cerebroplacental ratio in prediction of adverse perinatal outcome and fetal heart rate disturbances in uncomplicated pregnancy at 40 weeks and beyond 16.Vol.11(1),PP.142-148., 2015.

[47] A.A.Baschat \& U.Gembruch: The cerebroplacental Doppler ratio revisited.Ultrasound Obstet Gynecol.Vol. 21(2),PP. 124-7,Feb ,2003.

[48] R.Hershkovitz, J.C.P.Kingdom\&C.H.Rodeck, Fetal cerebral blood flow redistribution in late gestation: Identification of compromise in small fetuses with normal umbilical artery Doppler. Ultrasound Obstet Gynecol.Vol. 15,PP.209-212, 2000 
[49] G.Sterne, L.E. Shields \& T.J.Dubinsky, Abnormal fetal cerebral \& umbilical Doppler measurements in fetus with intrauterine growth restriction predicts the severity of perinatal morbidity. J Clin Ultrasound.Vol. 29,PP.146-151, 2001.

[50] P.Vergani, N.Roncaglia , A.Locatelli , C.Andreotti , Crippa I, Pezzullo JC, Ghidini A..: Antenatal predictors of neonatal outcome in fetal growth restriction with absent end-diastolic flow in the umbilical artery. Am J Obstet Gynecol. Vol.193,PP.1213-1218, 2005.

[51] M.Palacio, F.Figueras \& M.Lanna, Middle cerebral artery Doppler indices at different sites: prediction of umbilical cord gases in prolonged pregnancies. Ultrasound Obstet Gynecol.Vol. 24,PP.529-533, 2004.

[52] R.O.Bahado-Singh, E.Kovanchi, A.Jeffres, U.Oz, O.Deren, J.Copel, G.Mari, The Doppler cerebroplacental ratio and perinatal outcome in intrauterine growth restriction. Am J Obstet Gynecol. Vol. 180,PP. 250-256, 1999.

[53] P.A.Devine, L.A.Bracero, A.Lysikiewicz , R.Evans , S.Wo-mack, D.W.Byrne, Middle cerebral to umbilical artery Doppler ratio in postdate pregnancies. Obstet Gynecol.Vol. 84,PP.856-860, 1994.

[54] D.Gramellini,

M.Folli, S.Raboni, E.Vadora_, A.Merialdi, Cerebralumbilical Doppler ratio as predictor of adverse outcome. Obstet Gynecol.Vol. 79,PP.416-420, 1992.

[55] M.Susumu \& Masahiko. The Doppler cerebroplacental ratio predicts non-reassuring fetal status in intrauterine growth restriction fetuses at term. Journal of Obstetrics \& Gynecology, Japan Society of Obstetrics \& Gynecology,Vol.25,PP.78-82, 2011.

[56] S.Yoshimura, H. Masuzaki , K.Miura , H.Gotoh , T.Ishimaru, Fetal blood flow redistribution in term intrauterine growth retardation \& postnatal growth. International Journal of Obsterics \& Gynecology.Vol. 9,PP.38, 1998.

[57] U.Gupta, S.Chandra \& M.K.Narula, Value of middle cerebral artery to umbilical artery ratio by Doppler velocimetry in pregnancies beyond term Obstet Gynecol India. Vol. 56(1),PP.37-40, 2006. 